Original Article

\title{
Isolation and characterization of bacteria residing in the oral, gut, and fecal samples of different pheasant species
}

\author{
Isolamento e caracterização de bactérias residentes nas amostras orais, intestinais e \\ fecais de diferentes espécies de faisão
}

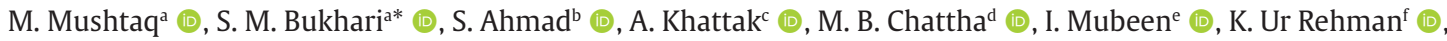

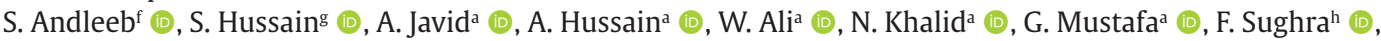 \\ M. J. Iqbali (D), M. Khalidj (D), M. M. Naeem ${ }^{\mathrm{k}}$ (D) and M. Inayat ${ }^{1}$ (D) \\ aUniversity of Veterinary and Animal Sciences, Faculty of Fisheries \& Wildlife, Department of Wildlife \& Ecology, Lahore, Pakistan \\ bUniversity of the Punjab, Department of Entomology, Lahore, Pakistan \\ 'Shaheed Benazir Bhutto Women University, Department of Bioinformatics, Peshawar, Pakistan \\ dUniversity of the Punjab, Faculty of Agricultural Sciences, Department of Agronomy, Lahore, Pakistan \\ ${ }^{e}$ Government College University, Department of Zoology, Lahore, Pakistan \\ ${ }^{\mathrm{f}} \mathrm{GC}$ Women University, Department of Environmental Sciences, Sialkot, Pakistan \\ ${ }^{g}$ Northeast Forestry University, College of Wildlife and Protected Area, Harbin, China \\ hUniversity of Education, Department of Zoology, Division of Science and Technology, Lahore, Pakistan \\ 'Bahauddin Zakariya University, Institute of Pure and Applied Biology (Zoology Division), Multan, Pakistan \\ ${ }^{\mathrm{j} E m e r s o n ~ U n i v e r s i t y ~ M u l t a n, ~ D e p a r t m e n t ~ o f ~ Z o o l o g y, ~ P u n j a b, ~ P a k i s t a n ~}$ \\ kInstitute of Engineering and Fertilizer Research, Department of Civil Engineering NFC, Faisalabad, Pakistan \\ 'University of Veterinary and Animal Sciences, Department of Fisheries and Aquaculture, Lahore, Pakistan
}

\begin{abstract}
There is a paucity of research conducted on microbial prevalence in pheasants. The microbiota of captive birds has zoonotic significance and must be characterize. Present study is therefore planned to assess the microbiota from oral, fecal and gut content of captive avian species. It will be helpful in characterization of harmful microbes. Different samples taken from oral, gut and feces of ring-necked pheasants (Phasianus colchicus), green pheasants (Phasianus versicolor), golden pheasant (Chrysolophus pictus) and silver pheasant (Lophura nycthemera). Samples were collected, diluted, and inoculated onto different agar plates (MacConkey, SS agar, MSA and nutrient agar) for cultivation of bacterial species. Colonies of E.coli, Staphylococcus spp. Brachyspira spp. and Campylobacter spp were observed based on colony morphology. Colony forming unit showed E. coli as frequently found bacteria in fecal, oral and gut contents of all the above pheasants. The overall significance difference was found among bacterial species of golden pheasants, green pheasant, ring-necked pheasant, and silver pheasants. It was concluded that E.coli is predominant isolated from heathy pheasants followed by Campylobacter, Staphylococcus and Brachyspira. Keywords: pheasants, biochemical characterization, fecal microbiota, prevalence, CFU method, diversity, microbiota.

Resumo

Há uma escassez de pesquisas realizadas sobre a prevalência microbiana em faisões. A microbiota de aves em cativeiro tem significado zoonótico e deve ser caracterizada. O presente estudo está, portanto, planejado para avaliar a microbiota do conteúdo oral, fecal e intestinal de espécies aviárias em cativeiro. Será útil na caracterização de micróbios nocivos. Diferentes amostras retiradas da boca, intestino e fezes de faisões de pescoço redondo (Phasianus colchicus), faisões verdes (Phasianus versicolor), faisões dourados (Chrysolophus pictus) e faisão prateado (Lophura nycthemera). As amostras foram coletadas, diluídas e inoculadas em diferentes placas de ágar (MacConkey, ágar SS, MSA e ágar nutriente) para o cultivo de espécies bacterianas. Colônias de E. coli, Staphylococcus spp., Brachyspira spp. e Campylobacter spp foram observados com base na morfologia da colônia. A unidade formadora de colônia mostrou E. coli como bactéria frequentemente encontrada no conteúdo fecal, oral e intestinal de todos os faisões acima. A diferença de significância geral foi encontrada entre as espécies bacterianas de faisões dourados, faisões verdes, faisões de pescoço anelado e faisões prateados. Verificou-se que a E.coli é predominantemente isolada de faisões saudáveis, seguida por Campylobacter, Staphylococcus e Brachyspira.
\end{abstract}

Palavras-chave: faisões, caracterização bioquímica, microbiota fecal, prevalência, método CFU, diversidade, microbiota.

*e-mail: mohsin.bukhari@uvas.edu.pk

Received: February 26, 2021 - Accepted: May 19, 2021 


\section{Introduction}

Birds have cosmopolitan distribution unveil morphological and ecological diversity and are more diverse in the Neotropics (Jenkins et al., 2013). The microbiomes of the gut play a major role in protection against pathogens, aid in digestion, hence affect the immunity and health of organisms. The abundance of microbiomes varies with species, captive status, sampling site, and diet (Gilbert et al., 2016; Waite and Taylor, 2014).

Microbiota is defined as the cannibalistic, coexisting microorganisms that reside inside the hosts without causing any harm (Sekirov et al., 2010). The gut microbiota plays a vital role in influencing physiology, immune system, and health. Microbes aid in the maintenance of important functions of the host such as help in digestion, synthesis of vitamins, defense, immune system, and organ development. The gut may inhabit thousands of species of microbes and be considered a densely populated natural environment. Frequently, microbiota may have an effect beyond the individual level and can distress mate choice (Sekirov et al., 2010).

The community of bacteria present in the gastrointestinal tract of birds is important to determine the host's health and physiology. For the understanding of the host microbiomes, different methods such as oral, fecal, and gut sampling have been developed to sample them from individuals. Mostly cloacal sampling was used in the past to sample microbiomes of birds and reptiles (Ganz et al., 2017). Cloacal sampling is extensively used as it is easy to perform and allows repeated sampling from the same individuals. Cloacal sampling has practical advantages over fecal sampling as fecal sampling is sometimes unreliable and provides potential problems in identifying sample ownership and time of defecation.

Microbiota diversity depends on species, age, diet, host site, and social climatic conditions. Pathogens of Galliformes include Brachyspira, Campylobacter jejuni, and Clostridium perfringens (Stanley et al., 2014; Vasai et al., 2014; Neo et al., 2013). Understanding of microbes and their relationship with the hosts is necessary to improve the health of host organisms (Gilbert et al., 2016; Roto et al., 2015) as investigation on Galliformes' caecal microbiomes is useful in understanding the sources of pathogenic bacteria and could be helpful in management practices of these taxa in captivity (Best et al., 2017).

Brachyspira hyodysenteriae is a parasite that resides in pigs, but it was also recorded in Mallard (Jansson et al., 2004). Levesque et al. (2000) recorded Campylobacter, Staphylococcus aureus, and Pseudomonas aeruginosa from the fecal droppings of the ring-billed gull. Campylobacter jejuni (50\%), E. coli (67\%), and Salmonella spp. were recorded from the gut contents of avian species (Fallacara et al., 2001).

The common pheasant (Phasianus colchicus) is a renowned game bird species and is mostly hunted throughout the world. In addition, pheasant's meat has high nutritive value, high-quality protein, and low in fat (Franco and Lorenzo, 2013). Previously, lactobacilli and enterococci were identified in the feces of pheasants but staphylococcus mostly essential microflora in pheasants (Lauková and Kandričáková, 2015). Methicillin-resistant staphylococcus (MRS) can be possible causative agents in pheasant's husbandry as their meat is used as a food risk for the health of consumers. Bacteriocins (enterocins) can be effectively used for husbandry in poultry hens, Japanese quails, ostriches, and rabbits. Their application is evaluated by EFSA and reported that they do not leave residues in meat (Lauková and Kandričáková, 2015).

The study of commercial birds describes that the microbiota varies according to change in environmental conditions of the commercial site, species of birds, diet, and host site (van der Wielen et al., 2002; Lu et al., 2003; Gong et al., 2007; Stanley et al., 2014; Vasaï et al., 2014; Roto et al., 2015).

Most of the bacteria linked with avian species are found in intestinal caeca because there is the relatively lower partial pressure of oxygen, low enzymatic activity as well as low bile salts concentrations that make a suitable environment for most of the bacterial species (Gabriel et al., 2006). The gut microbiome has exhibited an incredible effect on the fitness and appearance of adults by its influence on the structure of gut, nervous and immune system development, metabolism (Sharon et al., 2016). There is a paucity of research conducted on microbial prevalence in pheasants. The microbiota of captive birds has zoonotic significance and must be characterized. The present study is therefore planned to assess the microbiota from oral, fecal, and gut content of captive avian species. It will be helpful in the characterization of harmful microbes.

\section{Materials and Methods}

\subsection{Experimental site}

Pheasants such as ring-necked pheasants (Phasianus colchicus), green pheasants (Phasianus versicolor), golden pheasants ((Chrysolophus pictus), and silver pheasant (Lophura nycthemera) were maintained at Avian Conservation and Research Center, Department of Wildlife and Ecology, University of Veterinary and Animal Sciences, Ravi Campus, Pattoki, District Kasur, Pakistan.

\subsection{Sampling site and sample collection}

The oral, fecal, and gut samples of four pheasant species were taken and analyzed in the microbiology laboratory.

\subsection{Oral, fecal, and gut sampling}

Oral samples were taken by inserting a sterilized cotton swab in the oral cavity of the pheasant species. The gut microbiota was collected by dissecting the pheasant species and diluting the gut content in saline. Fresh fecal samples were collected from pheasants aseptically using forceps, preserved into sterile conical tubes with screw caps (Murphy et al., 2005). Samples were stored in sterile polythene bags and immediately brought to the laboratory for further processing.

\subsection{Homogenization and serial dilution of samples}

Two grams of fecal sample from each species were mixed with 200ul PBS solution. The sample was centrifuged at 
$5000 \mathrm{rpm} / \mathrm{m}$ until dilution became clear. Fecal sample, gut sample, and oral sample were serially diluted up to 6 folds and CFU was calculated by pour plate method.

\subsection{Cultivation and total viable count}

$1 \mathrm{~mL}$ sample of serially diluted up to $10^{-6}$ was taken and spread onto different agar plates like MacConkey, SS agar, MSA, and nutrient agar following the spread-plate method and was incubated at $37^{\circ} \mathrm{C}$ for 24 hours. The colony morphology of E. coli, Staphylococcus spp. Brachyspira spp. and Campylobacter spp. were observed. Pure cultures of each type of bacteria were obtained as per procedures described by OIE (2000). Bacteriological examinations were carried out using the standard method for aerobic bacteria (Waite and Taylor, 2014).

Enumeration of bacterial species per $\mathrm{mL}$ in original culture was calculated by multiplying the number of colonies counted by the dilution factor (Equation 1 ).

Number of cells per $\mathrm{mL}=$ Number of colonies $\times$ Reciprocal of the dilution factor

\subsection{Cell morphology, staining characteristics, and identification of organisms}

Among the various characteristics, cell morphology characteristics like shape (bacilli, spiral, filamentous) and arrangement of bacterial cells appeared into chains and clusters were observed under a compound microscope after staining.

Isolated organisms were subjected to various biochemical tests (lactose fermentation test, Oxidase test, Catalase test, Indole test, MR Test, Voges-Proskauer test, Motility, and Indole Urease test as per the standard methods described by Cheesbrough (1985).

\subsection{Statistical analysis}

Statistical analysis was performed by using SAS (version 9.1) to compare means by ANOVA at alpha value of 0.01 .

\section{Results}

The present study determined the prevalence along with the characterization of four important bacterial species from fecal, oral and gut content in four species of pheasants viz., ring-necked pheasants, green pheasants, golden pheasants and silver pheasants.

\subsection{Biochemical characterization of isolates from fecal, oral and gut contents}

The biochemical test with each isolate was performed. The results of the biochemical tests are shown in Tables 1, 2, 3 and 4 for the bacterial colonies isolated from golden, green, ring necked and silver pheasant, respectively.

Table 1. Biochemical Characterization of isolates from Fecal, Oral and Gut contents of Golden Pheasant.

\begin{tabular}{|c|c|c|c|c|c|c|c|c|c|c|c|c|}
\hline Test Performed & $f(E)$ & $\mathbf{f}(\mathbf{C})$ & $\mathbf{f}(B)$ & $\mathbf{f}(\mathbf{S})$ & $\mathbf{O}(\mathrm{E})$ & $\mathbf{O}(\mathrm{C})$ & $\mathbf{O}(\mathbf{B})$ & $\mathbf{O}(\mathbf{S})$ & $G(E)$ & $G(C)$ & G(B) & $\mathrm{G}(\mathbf{S})$ \\
\hline Catalase & + & - & + & + & + & + & + & + & + & + & + & + \\
\hline Oxidase & - & + & + & - & - & + & + & - & - & + & + & - \\
\hline Motility & + & + & $\mathrm{N} / \mathrm{A}$ & - & + & + & $\mathrm{N} / \mathrm{A}$ & - & + & + & $\mathrm{N} / \mathrm{A}$ & - \\
\hline Endospore staining & - & - & N/A & - & - & - & $\mathrm{N} / \mathrm{A}$ & - & - & - & $\mathrm{N} / \mathrm{A}$ & - \\
\hline Lactose Fermentation & + & - & $\mathrm{N} / \mathrm{A}$ & + & + & - & $\mathrm{N} / \mathrm{A}$ & + & + & - & $\mathrm{N} / \mathrm{A}$ & + \\
\hline Methyl Red & + & - & $\mathrm{N} / \mathrm{A}$ & + & + & - & $\mathrm{N} / \mathrm{A}$ & + & + & - & $\mathrm{N} / \mathrm{A}$ & + \\
\hline Voges-Proskauer & - & - & $\mathrm{N} / \mathrm{A}$ & + & - & - & $\mathrm{N} / \mathrm{A}$ & + & - & - & $\mathrm{N} / \mathrm{A}$ & + \\
\hline Indole & + & - & + & - & + & - & + & - & + & - & + & - \\
\hline Citrate & - & + & $\mathrm{N} / \mathrm{A}$ & + & - & + & $\mathrm{N} / \mathrm{A}$ & + & - & + & $\mathrm{N} / \mathrm{A}$ & + \\
\hline
\end{tabular}

$\mathrm{N} / \mathrm{A}=$ Did not show any result $; \mathrm{f}=$ fecal $; \mathrm{O}=$ oral $; \mathrm{G}=$ gut $; \mathrm{E}=\mathrm{E}$-Coli $; \mathrm{C}=$ Campylobacter $\mathrm{S}=$ Staphylococcus $; \mathrm{B}=$ Brachyspira .

Table 2. Biochemical Characterization of isolates from Fecal, Oral and Gut contents of Green Pheasant.

\begin{tabular}{|c|c|c|c|c|c|c|c|c|c|c|c|c|}
\hline Test Performed & $f(E)$ & $f(C)$ & $\mathbf{f}(\mathbf{B})$ & $\mathbf{f}(\mathbf{S})$ & $\mathbf{O}(\mathrm{E})$ & $\mathbf{O}(\mathrm{C})$ & $\mathbf{O}(\mathbf{B})$ & $\mathbf{O}(\mathbf{S})$ & $G(E)$ & $\mathbf{G}(\mathbf{C})$ & G(B) & $\mathbf{G}(\mathbf{S})$ \\
\hline Catalase & + & - & + & + & + & + & + & + & + & + & + & + \\
\hline Oxidase & - & + & + & - & - & + & + & - & - & + & + & - \\
\hline Motility & + & + & $\mathrm{N} / \mathrm{A}$ & - & + & + & $\mathrm{N} / \mathrm{A}$ & - & + & + & $\mathrm{N} / \mathrm{A}$ & - \\
\hline Endospore staining & - & - & $\mathrm{N} / \mathrm{A}$ & - & - & - & $\mathrm{N} / \mathrm{A}$ & - & - & - & $\mathrm{N} / \mathrm{A}$ & - \\
\hline Lactose Fermentation & + & - & $\mathrm{N} / \mathrm{A}$ & + & + & - & $\mathrm{N} / \mathrm{A}$ & + & + & - & $\mathrm{N} / \mathrm{A}$ & + \\
\hline Methyl Red & + & - & $\mathrm{N} / \mathrm{A}$ & + & + & - & $\mathrm{N} / \mathrm{A}$ & + & + & - & $\mathrm{N} / \mathrm{A}$ & + \\
\hline Voges-Proskauer & - & - & $\mathrm{N} / \mathrm{A}$ & + & - & - & $\mathrm{N} / \mathrm{A}$ & + & - & - & $\mathrm{N} / \mathrm{A}$ & + \\
\hline Indole & + & - & + & - & + & - & + & - & + & - & + & - \\
\hline Citrate & - & + & $\mathrm{N} / \mathrm{A}$ & + & - & + & $\mathrm{N} / \mathrm{A}$ & + & - & + & $\mathrm{N} / \mathrm{A}$ & + \\
\hline
\end{tabular}

$\mathrm{N} / \mathrm{A}=$ Did not show any result $; \mathrm{f}=$ fecal $; \mathrm{O}=$ oral $; \mathrm{G}=$ gut $; \mathrm{E}=\mathrm{E}-$ Coli $; \mathrm{C}=$ Campylobacter $; \mathrm{S}=$ Staphylococcus $; \mathrm{B}=$ Brachyspira . 
Table 3. Biochemical Characterization of isolates from Fecal, Oral and Gut contents of Ring-Necked Pheasant.

\begin{tabular}{|c|c|c|c|c|c|c|c|c|c|c|c|c|}
\hline Test Performed & $f(E)$ & $\mathbf{f}(\mathbf{C})$ & $f(B)$ & $\mathbf{f}(\mathbf{S})$ & $\mathbf{O}(\mathbf{E})$ & $\mathbf{O}(\mathrm{C})$ & $\mathbf{O}(\mathbf{B})$ & $\mathbf{O}(\mathbf{S})$ & $G(E)$ & $G(C)$ & $G(B)$ & $\mathbf{G}(\mathbf{S})$ \\
\hline Catalase & + & - & + & + & + & + & + & + & + & + & + & + \\
\hline Oxidase & - & + & + & - & - & + & + & - & - & + & + & - \\
\hline Motility & + & + & $\mathrm{N} / \mathrm{A}$ & - & + & + & $\mathrm{N} / \mathrm{A}$ & - & + & + & $\mathrm{N} / \mathrm{A}$ & - \\
\hline Endospore staining & - & - & $\mathrm{N} / \mathrm{A}$ & - & - & - & $\mathrm{N} / \mathrm{A}$ & - & - & - & $\mathrm{N} / \mathrm{A}$ & - \\
\hline Lactose Fermentation & + & - & $\mathrm{N} / \mathrm{A}$ & + & + & - & $\mathrm{N} / \mathrm{A}$ & + & + & - & $\mathrm{N} / \mathrm{A}$ & + \\
\hline Methyl Red & + & - & $\mathrm{N} / \mathrm{A}$ & + & + & - & $\mathrm{N} / \mathrm{A}$ & + & + & - & $\mathrm{N} / \mathrm{A}$ & + \\
\hline Voges-Proskauer & - & - & $\mathrm{N} / \mathrm{A}$ & + & - & - & $\mathrm{N} / \mathrm{A}$ & + & - & - & $\mathrm{N} / \mathrm{A}$ & + \\
\hline Indole & + & - & + & - & + & - & + & - & + & - & + & - \\
\hline Citrate & - & + & $\mathrm{N} / \mathrm{A}$ & + & - & + & $\mathrm{N} / \mathrm{A}$ & + & - & + & $\mathrm{N} / \mathrm{A}$ & + \\
\hline
\end{tabular}

$\mathrm{N} / \mathrm{A}=$ Did not show any result $\mathrm{f}=$ fecal; $\mathrm{O}=$ oral $; \mathrm{G}=$ gut $\mathrm{E}=\mathrm{E}$-Coli $; \mathrm{C}=$ Campylobacter $; \mathrm{S}=$ Staphylococcus $; \mathrm{B}=$ Brachyspira.

Table 4. Biochemical Characterization of isolates from Fecal, Oral and Gut contents of Silver Pheasant.

\begin{tabular}{|c|c|c|c|c|c|c|c|c|c|c|c|c|}
\hline Test Performed & $f(E)$ & $f(C)$ & $\mathbf{f}(\mathbf{B})$ & $\mathbf{f}(\mathbf{S})$ & $\mathbf{O}(\mathbf{E})$ & $\mathbf{O}(\mathrm{C})$ & $\mathbf{O}(\mathbf{B})$ & $\mathbf{O}(\mathbf{S})$ & $G(E)$ & $\mathbf{G}(\mathbf{C})$ & $\mathbf{G}(\mathbf{B})$ & $\mathbf{G}(\mathbf{S})$ \\
\hline Catalase & + & - & + & + & + & + & + & + & + & + & + & + \\
\hline Oxidase & - & + & + & - & - & + & + & - & - & + & + & - \\
\hline Motility & + & + & $\mathrm{N} / \mathrm{A}$ & - & + & + & $\mathrm{N} / \mathrm{A}$ & - & + & + & $\mathrm{N} / \mathrm{A}$ & - \\
\hline Endospore staining & - & - & $\mathrm{N} / \mathrm{A}$ & - & - & - & $\mathrm{N} / \mathrm{A}$ & - & - & - & $\mathrm{N} / \mathrm{A}$ & - \\
\hline Lactose Fermentation & + & - & $\mathrm{N} / \mathrm{A}$ & + & + & - & $\mathrm{N} / \mathrm{A}$ & + & + & - & $\mathrm{N} / \mathrm{A}$ & + \\
\hline Methyl Red & + & - & $\mathrm{N} / \mathrm{A}$ & + & + & - & $\mathrm{N} / \mathrm{A}$ & + & + & - & $\mathrm{N} / \mathrm{A}$ & + \\
\hline Voges-Proskauer & - & - & $\mathrm{N} / \mathrm{A}$ & + & - & - & $\mathrm{N} / \mathrm{A}$ & + & - & - & $\mathrm{N} / \mathrm{A}$ & + \\
\hline Indole & + & - & + & - & + & - & + & - & + & - & + & - \\
\hline Citrate & - & + & $\mathrm{N} / \mathrm{A}$ & + & - & + & $\mathrm{N} / \mathrm{A}$ & + & - & + & $\mathrm{N} / \mathrm{A}$ & + \\
\hline
\end{tabular}

$\mathrm{N} / \mathrm{A}=$ Did not show any result $; \mathrm{f}=$ fecal $; \mathrm{O}=$ oral $; \mathrm{G}=$ gut $; \mathrm{E}=\mathrm{E}$-Coli $; \mathrm{C}=$ Campylobacter $; \mathrm{S}=$ Staphylococcus $; \mathrm{B}=$ Brachyspira.

\subsection{Assessment of microbiota from fecal, gut and oral} samples of Golden Pheasants

The mean prevalence of E.coli, Campylobacter, Staphylococcus and Brachyspira in fecal samples were $2.81 \times 10^{9} \pm 0.78,1.83 \times 10^{9} \pm 0.51,8.81 \times 10^{8} \pm 2.57$ and $9.57 \times 10^{8}$ \pm 2.50 CFUs, respectively. In case of gut sample, the mean prevalence of E.coli, Campylobacter, Staphylococcus and Brachyspira were $1.47 \times 10^{9} \pm 0.40,4.88 \times 10^{8} \pm 1.217 .77 \times 10^{8}$ \pm 1.82 , and $2.80 \times 10^{8} \pm 0.04$ CFUs, respectively. Similarly, in oral samples the mean prevalence of E.coli, Campylobacter, Staphylococcus and Brachyspira were $4.83 \times 10^{8} \pm 1.13$, $8.24 \times 10^{8} \pm 2.40,3.65 \times 10^{8} \pm 1.43$ and $5.78 \times 10^{7} \pm 2.80$ CFUs, respectively. Table 5 representing significant difference between microbiota of golden pheasants from oral, gut and fecal samples at 99\% level of significance.

\subsection{Assessment of microbiota from fecal, gut and oral samples of Green Pheasants}

Fecal sample showed the mean prevalence $2.73 \times 10^{9}$ $\pm 0.76,8.92 \times 10^{8} \pm 2.67,9.21 \times 10^{8} \pm 2.65$ and $1.79 \times 10^{8} \pm$ 0.84 CFUs for E.coli, Campylobacter, Staphylococcus and Brachyspira, respectively. Mean prevalence of E.coli, Campylobacter, Staphylococcus and Brachyspira was $1.46 \times 10^{9}$ $\pm 0.41,5.59 \times 10^{8} \pm 1.45,6.17 \times 10^{8} \pm 1.84$ and $1.05 \times 10^{8} \pm$
0.07CFUs in gut samples. While oral samples showed mean prevalence of E.coli, Campylobacter, Staphylococcus and Brachyspira, $7.52 \times 10^{8} \pm 2.29,4.25 \times 10^{8} \pm 1.20,4.42 \times 10^{8}$ \pm 1.30 and $6.94 \times 10^{6} \pm 1.79$ CFUs, respectively. Table 5 representing significant difference between microbiota of green pheasants from oral, gut and fecal samples at 99\% level of significance.

\subsection{Assessment of microbiota from fecal, gut and oral samples of Ring-Necked Pheasants}

Mean prevalence of E.coli, Campylobacter, Staphylococcus and Brachyspira was $2.13 \times 10^{9} \pm 0.61,5.17 \times 10^{8} \pm 1.36$, $4.52 \times 10^{8} \pm 1.22$ and $1.52 \times 10^{8} \pm 0.43$ CFUs, respectively of fecal samples. Mean prevalence of E.coli, Campylobacter, Staphylococcus and Brachyspira was $1.47 \times 10^{9} \pm 0.40$, $4.88 \times 10^{8} \pm 1.21,7.77 \times 10^{8} \pm 1.82$ and $2.80 \times 10^{8} \pm 0.04$ CFUs respectively in gut samples. Similarly, mean prevalence E.coli, Campylobacter, Staphylococcus and Brachyspira in oral samples was $4.83 \times 10^{8} \pm 1.13,8.24 \times 10^{8} \pm 2.40,3.65 \times 10^{8}$ \pm 1.43 and $5.78 \times 10^{7} \pm 2.80$ CFUs, respectively. Table 5 representing significant difference between microbiota of Ring-Necked pheasants from oral, gut and fecal samples at $99 \%$ level of significance. 
3.5. Assessment of microbiota from fecal, gut and oral samples of Silver Pheasants

E.coli, Campylobacter, Staphylococcus and Brachyspira mean prevalence was $2.55 \times 10^{9} \pm 0.70,2.04 \times 10^{8} \pm 0.05$,
$1.94 \times 10^{8} \pm 0.03$ and $1.76 \times 10^{8} \pm 0.10$ CFUs, respectively of fecal samples. The mean prevalence of E.coli, Campylobacter, Staphylococcus and Brachyspira was $5.43 \times 10^{8} \pm 1.52$, $1.37 \times 10^{8} \pm 0.07,1.08 \times 10^{8} \pm 0.07$ and $1.22 \times 10^{8} \pm 0.056$ CFUs

Table 5. Total variable counts expressed as CFUs for each bacterial species from Oral, Gut and Feces of Silver, Golden, Ring neck and Green pheasants, respectively.

\begin{tabular}{|c|c|c|c|c|c|c|c|}
\hline \multirow[b]{2}{*}{ Bird species } & \multirow[b]{2}{*}{ parameters } & \multirow{2}{*}{$\begin{array}{l}\text { Bacteria } \\
\text { species }\end{array}$} & \multirow{2}{*}{$\begin{array}{c}\text { Enumeration } \\
\text { of total } \\
\text { microbial } \\
\text { count }\end{array}$} & P value & \multirow{2}{*}{$\begin{array}{c}\text { Prevalence } \\
\text { (Mean } \pm \text { SEM) }\end{array}$} & \multirow{2}{*}{$\begin{array}{l}\text { P value } \\
\text { (Species) }\end{array}$} & \multirow{2}{*}{$\begin{array}{c}\text { P value } \\
\text { (Species) }\end{array}$} \\
\hline & & & & (Species) & & & \\
\hline \multirow{12}{*}{$\begin{array}{l}\text { Golden pheasant } \\
\text { (Mean } \pm \text { SEM })\end{array}$} & \multirow[t]{4}{*}{ Oral } & E-Coli & $4.83 \times 10^{8} \pm 1.13$ & \multirow[t]{4}{*}{$0.0102^{*}$} & \multirow{4}{*}{$\begin{array}{l}4.10 \times 10^{7} \pm \\
8.42^{\mathrm{B}}\end{array}$} & \multirow[t]{12}{*}{$.0001^{*}$} & \\
\hline & & Campylobacter & $8.24 \times 10^{8} \pm 2.40$ & & & & \\
\hline & & Staphylococcus & $3.65 \times 10^{8} \pm 1.43$ & & & & \\
\hline & & Brachyspira & $5.78 \times 10^{7} \pm 2.80$ & & & & \\
\hline & \multirow[t]{4}{*}{ Fecal } & E-Coli & $2.81 \times 10^{9} \pm 0.78$ & \multirow[t]{4}{*}{$0.0332^{*}$} & \multirow{4}{*}{$\begin{array}{c}1.62 \times 10^{9} \pm 0 \\
27^{\mathrm{A}}\end{array}$} & & \\
\hline & & Campylobacter & $1.83 \times 10^{9} \pm 0.51$ & & & & \\
\hline & & Staphylococcus & $8.81 \times 10^{8} \pm 2.57$ & & & & \\
\hline & & Brachyspira & $9.57 \times 10^{8} \pm 2.50$ & & & & \\
\hline & \multirow[t]{4}{*}{ Gut } & E-Coli & $1.47 \times 10^{9} \pm 0.40$ & \multirow[t]{4}{*}{$0.0046^{*}$} & \multirow{4}{*}{$\begin{array}{c}7.55 \times 10^{8} \pm \\
1.32^{\mathrm{B}}\end{array}$} & & \\
\hline & & Campylobacter & $4.88 \times 10^{8} \pm 1.21$ & & & & \\
\hline & & Staphylococcus & $7.77 \times 10^{8} \pm 1.82$ & & & & \\
\hline & & Brachyspira & $2.80 \times 10^{8} \pm 0.04$ & & & & \\
\hline \multirow{12}{*}{$\begin{array}{c}\text { Ring neck } \\
\text { pheasant (Mean } \\
\pm \text { SEM) }\end{array}$} & \multirow[t]{4}{*}{ Oral } & E-Coli & $4.83 \times 10^{8} \pm 1.13$ & \multirow[t]{4}{*}{$0.0102^{*}$} & \multirow{4}{*}{$\begin{array}{c}4.32 \times 10^{8} \pm \\
5.38^{\mathrm{B}}\end{array}$} & \multirow[t]{12}{*}{0.1423} & \multirow[t]{12}{*}{$0.0011^{*}$} \\
\hline & & Campylobacter & $8.24 \times 10^{8} \pm 2.40$ & & & & \\
\hline & & Staphylococcus & $3.65 \times 10^{8} \pm 1.43$ & & & & \\
\hline & & Brachyspira & $5.78 \times 10^{7} \pm 2.80$ & & & & \\
\hline & \multirow[t]{4}{*}{ Fecal } & E-Coli & $2.13 \times 10^{9} \pm 0.61$ & \multirow[t]{4}{*}{$0.0004^{*}$} & $8.14 \times 10^{8} \pm$ & & \\
\hline & & Campylobacter & $5.17 \times 10^{8} \pm 1.36$ & & $12.54^{\mathrm{A}}$ & & \\
\hline & & Staphylococcus & $4.52 \times 10^{8} \pm 1.22$ & & & & \\
\hline & & Brachyspira & $1.52 \times 10^{8} \pm 0.43$ & & & & \\
\hline & Gut & E-Coli & $1.47 \times 10^{9} \pm 0.40$ & $0.0046^{*}$ & $7.55 \times 10^{8} \pm$ & & \\
\hline & & Campylobacter & $4.88 \times 10^{8} \pm 1.21$ & & $8.35^{\mathrm{B}}$ & & \\
\hline & & Staphylococcus & $7.77 \times 10^{8} \pm 1.82$ & & & & \\
\hline & & Brachyspira & $2.80 \times 10^{8} \pm 0.04$ & & & & \\
\hline Green pheasant & Oral & E-Coli & $7.52 \times 10^{8} \pm 2.29$ & $0.0202^{*}$ & $4.30 \times 10^{8} \pm$ & $0.0041^{*}$ & \\
\hline$($ Mean \pm SEM $)$ & & Campylobacter & $4.25 \times 10^{8} \pm 1.20$ & & $0.81^{\mathrm{B}}$ & & \\
\hline & & Staphylococcus & $4.42 \times 10^{8} \pm 1.30$ & & & & \\
\hline & & Brachyspira & $6.94 \times 10^{6} \pm 1.79$ & & & & \\
\hline & Fecal & E-Coli & $2.73 \times 10^{9} \pm 0.76$ & $0.0012^{*}$ & $1.18 \times 10^{10} \pm$ & & \\
\hline & & Campylobacter & $8.92 \times 10^{8} \pm 2.67$ & & $0.02^{\mathrm{A}}$ & & \\
\hline & & Staphylococcus & $9.21 \times 10^{8} \pm 2.65$ & & & & \\
\hline & & Brachyspira & $1.79 \times 10^{8} \pm 0.84$ & & & & \\
\hline & Gut & E-Coli & $1.46 \times 10^{9} \pm 0.41$ & $0.0027^{*}$ & $6.88 \times 10^{8} \pm$ & & \\
\hline & & Campylobacter & $5.59 \times 10^{8} \pm 1.45$ & & $1.39^{\mathrm{B}}$ & & \\
\hline & & Staphylococcus & $6.17 \times 10^{8} \pm 1.84$ & & & & \\
\hline & & Brachyspira & $1.05 \times 10^{8} \pm 0.07$ & & & & \\
\hline
\end{tabular}

SEM = Standard Error Mean; “*” shows P value $<0.01=$ significant at $1 \%$ level of significant. 
Table 5. Continued...

\begin{tabular}{|c|c|c|c|c|c|c|c|}
\hline \multirow{2}{*}{ Bird species } & \multirow{2}{*}{ parameters } & \multirow{2}{*}{$\begin{array}{l}\text { Bacteria } \\
\text { species }\end{array}$} & \multirow{2}{*}{$\begin{array}{c}\text { Enumeration } \\
\text { of total } \\
\text { microbial } \\
\text { count }\end{array}$} & P value & \multirow{2}{*}{$\begin{array}{c}\text { Prevalence } \\
(\text { Mean } \pm \text { SEM })\end{array}$} & \multirow{2}{*}{$\begin{array}{l}\text { P value } \\
\text { (Species) }\end{array}$} & \multirow{2}{*}{$\begin{array}{r}\text { P value } \\
\text { (Species }\end{array}$} \\
\hline & & & & (Species) & & & \\
\hline \multirow{12}{*}{$\begin{array}{c}\text { Silver pheasant } \\
(\text { Mean } \pm \text { SEM) }\end{array}$} & \multirow[t]{4}{*}{ Oral } & E-Coli & $4.23 \times 10^{8} \pm 1.19$ & $0.0002^{*}$ & \multirow{4}{*}{$\begin{array}{c}1.63 \times 10^{8} \pm \\
0.37^{\mathrm{B}}\end{array}$} & \multirow[t]{12}{*}{$0.0104^{*}$} & \\
\hline & & Campylobacter & $8.52 \times 10^{7} \pm 0.55$ & & & & \\
\hline & & Staphylococcus & $7.54 \times 10^{7} \pm 0.47$ & & & & \\
\hline & & Brachyspira & $6.86 \times 10^{7} \pm 0.18$ & & & & \\
\hline & \multirow[t]{4}{*}{ Fecal } & E-Coli & $2.55 \times 10^{9} \pm 0.70$ & $<.0001^{*}$ & \multirow{4}{*}{$\begin{array}{c}7.82 \times 10^{8} \pm \\
2.35^{\mathrm{A}}\end{array}$} & & \\
\hline & & Campylobacter & $2.04 \times 10^{8} \pm 0.05$ & & & & \\
\hline & & Staphylococcus & $1.94 \times 10^{8} \pm 0.03$ & & & & \\
\hline & & Brachyspira & $1.76 \times 10^{8} \pm 0.10$ & & & & \\
\hline & \multirow[t]{4}{*}{ Gut } & E-Coli & $5.43 \times 10^{8} \pm 1.52$ & $0.0005^{*}$ & \multirow{4}{*}{$\begin{array}{c}2.27 \times 10^{8} \pm \\
0.46^{\mathrm{B}}\end{array}$} & & \\
\hline & & Campylobacter & $1.37 \times 10^{8} \pm 0.07$ & & & & \\
\hline & & Staphylococcus & $1.08 \times 10^{8} \pm 0.07$ & & & & \\
\hline & & Brachyspira & $1.22 \times 10^{8} \pm 0.056$ & & & & \\
\hline
\end{tabular}

SEM = Standard Error Mean; “*” shows P value $<0.01=$ significant at $1 \%$ level of significant.

respectively in gut samples. While in case of oral sample the mean prevalence of E.coli, Campylobacter, Staphylococcus and Brachyspira was $4.23 \times 10^{8} \pm 1.19,8.52 \times 10^{7} \pm 0.55$, $7.54 \times 10^{7} \pm 0.47$ and $6.86 \times 10^{7} \pm 0.18$, respectively. Table 5 representing significant difference between microbiota of Silver pheasants from oral, gut and fecal samples at a 99\% level of significance.

\section{Discussion}

In all the isolated bacterial species, the most prevalent species of bacteria was E.coli regardless of type of pheasant species. E.coli is naturally present in fecal sample of all the birds (Sarker et al., 2012), cattle and even the human. Total viable count was expressed as CFU and it showed mean \pm SD of E.coli colonies in golden pheasant as follows: oral sample showed $7.52 \times 10^{8} \pm 2.29$, fecal sample $2.73 \times 10^{9}$ \pm 0.76 and gut sample $1.47 \times 10^{9} \pm 0.40 \mathrm{CFU} /$ gram of the sample While, in case of prevalence of Campylobacter CFU/g of each sample were as follows: oral sample showed $8.24 \times 10^{8} \pm 2.40$, fecal sample showed $1.83 \times 10^{9} \pm 0.51$ and gut sample showed $4.88 \times 10^{8} \pm 1.21 \mathrm{CFU} / \mathrm{g}$ of the sample. In case of Campylobacter, again the fecal sample showed a higher prevalence rate. Staphylococcus was present in oral, fecal and gut sample as $3.65 \times 10^{8} \pm 1.43,8.81 \times 10^{8} \pm$ 2.5 and $7.77 \times 10^{8} \pm 1.82 \mathrm{CFU} / \mathrm{g}$ of the sample respectively. Brachyspira was present in oral, fecal and gut sample $5.78 \times 10^{7} \pm 2.80,9.57 \times 10^{8} \pm 2.50$ and $2.80 \times 10^{8} \pm 0.04 \mathrm{CFU} / \mathrm{g}$ of sample, respectively. In recent study, it was concluded that in golden pheasant the fecal sample harbors the greatest number of bacteria in it. As statistically, mean for total number of bacterial colonies isolated from fecal sample is $1.62 \times 10^{9} \pm 0.2$, which was significantly greater than the number of colonies isolated from the oral and gut samples as shown in Table 5.
In green pheasant, the E.coli colonies isolated from oral sample were $7.52 \times 10^{8} \pm 2.29 \mathrm{CFU} / \mathrm{g}$ of the sample, $2.73 \times 10^{9} \pm 0.76$ no. of E.coli CFU/g of the oral sample and in gut sample the colonies of E.coli isolated were $1.46 \times 10^{9}$ \pm 0.41 . Campylobacter showed $4.25 \times 10^{8} \pm 1.20,8.92 \times 10^{8} \pm$ 2.67 and $5.59 \times 10^{8} \pm 1.45 \mathrm{CFU} /$ gram of the oral, fecal and gut sample, respectively. Again, fecal sample showed the higher number of Campylobacter. In case of Staphylococcus and Brachyspira fecal sample showed higher number $9.21 \times 10^{8}$ $\pm 2.65 \mathrm{CFU} / \mathrm{g}$ with $1.79 \times 10^{8} \pm 0.84 \mathrm{CFU} / \mathrm{g}$ respectively.

In ring-necked pheasant, E.coli prevailed more in fecal sample than oral and gut sample. While the Campylobacter $\mathrm{CFU} / \mathrm{g}$ of oral sample was $8.24 \times 10^{8} \pm 2.40$ that is more than the CFU of Campylobacter in fecal and gut sample and Brachyspira was more in number in gut samples. The bacterial species in each sample were significantly different as the P-value $<0.05$ for each sample. Type of the diet and season could be the reason for this variation of bacterial species in different sample of the same species.

In the fecal sample of silver pheasant, E.coli was the most dominant than any other sample, Campylobacter showed more prevalence in oral sample and Staphylococcus showed more prevalence in fecal material in CFU/g. Brachyspira was more in fecal sample than oral and gut sample.

During this study, the most prevalent bacteria was E.coli, irrespective to type of sample and species and these findings correlate with the findings of Sarker et al. (2012) who collected 72 total samples of oral, gut and feces and found all sample positive for E.coli and in cloacal swab no Staphylococcus was isolated, while, $20 \%$ of the total samples were found positive for this bacteria. Conclusion of recent research supports Jahan et al. (2018) who found that ostrich fecal sample harbors more bacteria than the or pharyngeal sample and cloacal swab sample. The study amid to check the number of viable bacteria from oral, gut, and fecal samples. 
Selective media and biochemical tests were used for the isolation and identification of bacterial species which were previously suggested by Buxton and Fraser (1977), Cowan (1985) and Cheesbrough (1985). Biochemical characterization of isolates of bacterial species show positive and negative results against different tests. Species that did not show any result, were assigned as not applicable for certain test isolates for pheasants. The biochemical properties exposed by the isolates in this study are similar to reports of Buxton and Fraser (1977) and Cheesbrough (1985).

\section{Conclusion}

Most prevalent bacterial isolate, in the present study, among E.coli, Staphylococcus spp. Brachyspira spp. and Campylobacter spp was E.coli. It is concluded from the study that the shedding of pathogens was more frequent through feces. Fecal material could be a potential source of zoonotic pathogen carrier and must be managed and thrown away safely from aviaries and farms where birds like pheasants have been harbored.

\section{Acknowledgements}

This work was supported from the higher Education Commission, Pakistan through their start up research grant program (Grant No. SRGP1663).

\section{References}

BEST, A.A., PORTER, A.L., FRALEY, S.M. and FRALEY, G.S., 2017. Characterization of gut microbiome dynamics in developing pekin ducks and impact of management system. Frontiers in Microbiology, vol. 7, pp. 2125. http://dx.doi.org/10.3389/ fmicb.2016.02125. PMid:28101086.

BUXTON, A. and FRASER, G., 1977. Animal microbiology. Oxford: Blackwell Scientific Publications, vol. 1, pp. 85-110.

CHEESBROUGH, M., 1985. Medical laboratory manual for tropical countries. 1st ed. London: English Language Book Society, vol. 2. Microbiology, pp. 400-480.

COWAN, S.T., 1985. Cowan and Steel's manual for the identification medical bacteria. 2nd ed. Cambridge: Cambridge University Press, pp. 96-98.

FALLACARA, D.M., MONAHAN, C.M., MORISHITA, T.Y. and WACK, R.F., 2001. Fecal shedding and antimicrobial susceptibility of selected bacterial pathogens and a survey of intestinal parasites in free-living waterfowl. Avian Diseases, vol. 45, no. 1, pp. 128-135. http://dx.doi.org/10.2307/1593019. PMid:11332473.

FRANCO, D. and LORENZO, J.M., 2013. Meat quality and nutritional composition of pheasants Phasianus colchicus reared in an extensive system. British Poultry Science, vol. 54, no. 5, pp. 594-602. http://dx.doi.org/10.1080/00071668.2013.828195. PMid:24098978

GABRIEL, I., LESSIRE, M., MALLET, S. and GUILLOT, J.F., 2006. Microflora of the digestive tract: critical factors and consequences for poultry. World's Poultry Science Journal, vol. 62, pp. 499-511.
GANZ, H.H., DOROUD, L., FIRL, A.J., HIRD, S.M., EISEN, J.A. and BOYCE, W.M., 2017. Community-level differences in the microbiome of healthy wild mallards and those infected by influenza viruses. mSystems, vol. 2, no. 1, e00188-16. http://dx.doi.org/10.1128/ mSystems.00188-16. PMid:28293681.

GILBERT, J.A., QUINN, R.A., DEBELIUS, J., XU, Z.Z., MORTON, J., GARG, N., JANSSON, J.K., DORRESTEIN, P.C. and KNIGHT, R., 2016. Microbiome-wide association studies link dynamic microbial consortia to disease. Nature, vol. 535, no. 7610, pp. 94-103. http://dx.doi.org/10.1038/nature18850. PMid:27383984.

GONG, J., SI, W., FORSTER, R.J., HUANG, R., YU, H., YIN, Y., YANG, C. and HAN, Y., 2007. 16S rRNA gene-based analysis of mucosaassociated bacterial community and phylogeny in the chicken gastrointestinal tracts: from crops to ceca. FEMS Microbiology Ecology, vol. 59, no. 1, pp. 147-157. http://dx.doi.org/10.1111/ j.1574-6941.2006.00193.x. PMid:17233749.

JAHAN, I., RUMI, N.A., HOSSAIN, M.K., RAHMAN, M.S., FAKHRUZZAMAN, M., AKTER, S. and MIAH, A.G., 2018. Microbial assessment of different samples of ostrich Struthio camelus and determination of antimicrobial susceptibility profiles of the isolated bacteria. Asian Journal of Medical and Biological Research, vol.34, no. 4, pp. 437-445. http://dx.doi.org/10.3329/ ajmbr.v3i4.35334.

JANSSON, D.S., JOHANSSON, K.E., OLOFSSON, T., RÅSBÄCK, T., VÅGSHOLM, I., PETTERSSON, B., GUNNARSSON, A. and FELLSTRÖM, C., 2004. Brachyspira hyodysenteriae and other strongly beta-haemolytic and indole-positive spirochaetes isolated from mallards Anas platyrhynchos. Journal of Medical Microbiology, vol. 53, no. Pt 4, pp. 293-300. http://dx.doi. org/10.1099/jmm.0.05488-0. PMid:15017285.

JENKINS, C.N., PIMM, S.L. and JOPPA, L.N., 2013. Global patterns of terrestrial vertebrate diversity and conservation. Proceedings of the National Academy of Sciences of the United States of America, vol. 110, no. 28, pp. E2602-E2610. http://dx.doi.org/10.1073/ pnas.1302251110. PMid:23803854.

LAUKOVÁ, A. and KANDRIČÁKOVÁ, A., 2015. Staphylococci detected in faecal samples of common pheasants and their relation to enterocins. International Journal of Current Microbiology and Applied Sciences, vol. 4, pp. 788-797.

LEVESQUE, B., BROUSSEAU, P., BERNIER, F., DEWAILLY, E. and JOLY, J., 2000. Study of the bacterial content of ring-billed gull droppings in relation to recreational water quality. Water Research, vol.34, no.4, pp. 1089-1096. http://dx.doi.org/10.1016/ S0043-1354(99)00266-3.

LU, J., IDRIS, U., HARMON, B., HOFACRE, C., MAURER, J.J. and LEE, M.D., 2003. Diversity and succession of the intestinal bacterial community of the maturing broiler chicken. Applied and Environmental Microbiology, vol. 69, no. 11, pp.6816-6824. http:// dx.doi.org/10.1128/AEM.69.11.6816-6824.2003. PMid:14602645.

MURPHY, J., DEVANE, M.L., ROBSON, B. and GILPIN, B.J., 2005. Genotypic characterization of bacteria cultured from duck faeces. Journal of Applied Microbiology, vol. 99, no. 2, pp. 301-309. http:// dx.doi.org/10.1111/j.1365-2672.2005.02590.x. PMid:16033461.

NEO, E., LA, T., PHILLIPS, N.D., ALIKANI, M.Y. and HAMPSON, D.J., 2013. The pathogenic intestinal spirochaete Brachyspira pilosicoli forms a diverse recombinant species demonstrating some local clustering of related strains and potential for zoonotic spread. Gut Pathology, vol. 5, no. 1, pp. 24-28. http://dx.doi. org/10.1186/1757-4749-5-24. PMid:23957888.

OFFICE INTERNATIONAL DES EPIZOOTIES - OIE. 2000. Manual of standards for diagnostics tests and vaccines. Paris: OIE.

ROTO, S.M., RUBINELLI, P.M. and RICKE, S.C., 2015. An introduction to the avian gut microbiota and the effects of yeast-based 
prebiotic-type compounds as potential feed additives. Frontiers in Veterinary Science, vol. 2, pp. 28. http://dx.doi.org/10.3389/ fvets.2015.00028. PMid:26664957.

SARKER, M.A., JAHAN, M., PARVIN, M.N., MALEK, M.A. and HOSSAIN, M.T., 2012. Identification of bacterial flora isolated from apparently healthy water birds of Dhaka Zoo of Bangladesh. Bhikhabhai Jivabhai Vanijya Mahavidyalaya, vol. 101, no. 2, pp. 21-26.

SEKIROV, I., RUSSELL, S.L., ANTUNES, L.C. and FINLAY, B.B., 2010. Gut microbiota in health and disease. Physiological Reviews, vol. 90, no. 3, pp. 859-904.

SHARON, G., SAMPSON, T.R., GESCHWIND, D.H. and MAZMANIAN, S.K., 2016. The central nervous system and the gut microbiome. Cell, vol. 167, no. 4, pp. 915-932. http://dx.doi.org/10.1016/j. cell.2016.10.027. PMid:27814521.

STANLEY, D., HUGHES, R.J. and MOORE, R.J., 2014. Microbiota of the chicken gastrointestinal tract: influence on health, productivity, and disease. Applied Microbiology and Biotechnology, vol. 98, no. 10, pp. 4301-4310. http://dx.doi.org/10.1007/s00253-0145646-2. PMid:24643736.

VAN DER WIELEN, P.W., KEUZENKAMP, D.A., LIPMAN, L.J., VAN KNAPEN, F. and BIESTERVELD, S., 2002. Spatial and temporal variation of the intestinal bacterial community in commercially raised broiler chickens during growth. Microbial Ecology, vol. 44, no. 3, pp. 286-293. http://dx.doi.org/10.1007/s00248-0022015-y. PMid:12219265.

VASAÏ, F., BRUGIRARD RICAUD, K., BERNADET, M.D., CAUQUIL, L., BOUCHEZ, O., COMBES, S. and DAVAIL, S., 2014. Overfeeding and genetics affect the composition of intestinal microbiota in Anas platyrhynchos Pekin and Cairina moschata Muscovy ducks. FEMS Microbiology Ecology, vol. 87, no. 1, pp. 204-216. http://dx.doi.org/10.1111/1574-6941.12217. PMid:24102552.

WAITE, D.W. and TAYLOR, M.W., 2014. Characterizing the avian gut microbiota: membership, driving influences, and potential function. Frontiers in Microbiology, vol. 5, pp. 223. http://dx.doi. org/10.3389/fmicb.2014.00223. PMid:24904538. 